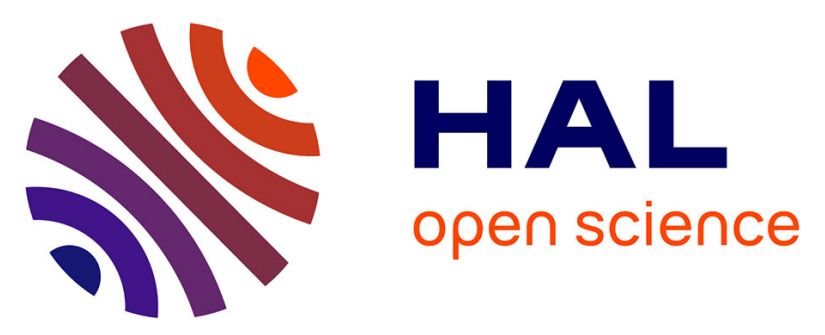

\title{
Endopolygalacturonases reveal molecular features for processivity pattern and tolerance towards acetylated pectin
}

\author{
Gwenaëlle André-Leroux, Dominique D. Tessier, Estelle Bonnin
}

\section{To cite this version:}

Gwenaëlle André-Leroux, Dominique D. Tessier, Estelle Bonnin. Endopolygalacturonases reveal molecular features for processivity pattern and tolerance towards acetylated pectin. Biochimica et Biophysica Acta Proteins and Proteomics, 2009, 1794 (1), pp.5-13. 10.1016/j.bbapap.2008.09.004 . hal-02660618

\section{HAL Id: hal-02660618 \\ https://hal.inrae.fr/hal-02660618}

Submitted on 30 May 2020

HAL is a multi-disciplinary open access archive for the deposit and dissemination of scientific research documents, whether they are published or not. The documents may come from teaching and research institutions in France or abroad, or from public or private research centers.
L'archive ouverte pluridisciplinaire HAL, est destinée au dépôt et à la diffusion de documents scientifiques de niveau recherche, publiés ou non, émanant des établissements d'enseignement et de recherche français ou étrangers, des laboratoires publics ou privés.

\section{다(1)(2)}

Distributed under a Creative Commons Attribution - ShareAlike| 4.0 International 


\title{
Endopolygalacturonases reveal molecular features for processivity pattern and tolerance towards acetylated pectin
}

\author{
G. André-Leroux ${ }^{\mathrm{a}}$, D. Tessier ${ }^{\mathrm{b}}$, E. Bonnin ${ }^{\mathrm{b}, *}$ \\ a Institut Pasteur, Unité de Biochimie Structurale, 25 rue du Dr Roux, 75724 Paris Cedex 15, France \\ b INRA, UR-1268 Biopolymères Interactions Assemblages, BP 71627, 44316 Nantes Cedex 3, France
}

\section{A R T I C L E I N F O}

\section{Article history:}

Received 30 January 2008

Received in revised form 1 September 2008

Accepted 6 September 2008

Available online 25 September 2008

\section{Keywords:}

Acetylated homogalacturonan

Tolerance profile

Processivity pattern

Oligogalacturonates docking

Normal mode analysis

\begin{abstract}
A B S T R A C T
Endopolygalacturonases (EndoPGs) hydrolyse the 1-4 linkages between two $\alpha$-D-galacturonic acids (GalA) of the smooth homogalacturonan regions of pectin. GalA may be methyl-esterified on the carboxylic group and acetyl-esterified on the hydroxylic groups. EndoPG activity most often decreases with such increasing degree of substitution. In this paper, we used bioinformatics and molecular modelling technics to explain the tolerance profile at the molecular scale and processivity scheme of three endoPGs with respect to acetylated pectin substrate; the first two enzymes originate from Aspergillus niger (AnPGI and AnPGII) and the third from Fusarium moniliforme (FmPG). Partly acetylated and methylated homogalacturonan fragments in complex with the three PGs were successively modelled in silico. The amino acid residues involved in substrate binding were identified for each enzyme. Similarly, the docking pattern of the differently decorated oligomers in the catalytic groove was individually characterized for each enzyme. This work shows full agreement with our previous extensive mass spectrometry analysis of the hydrolytic products that established distinct tolerance profiles for the three endoPGs and earlier work that ascertained processivity, specifically for AnPGI. In our previous work, AnPGI was shown to be the most powerful enzyme among the three enzymes with an enhanced tolerance towards 02 - and 03-acetylated substrates. We report here amino acids of AnPGI that are unique in binding the pectin backbone and that are identified as possibly crucial for its specificity, namely S191 ${ }_{A n P G /} / \mathrm{D} 240_{\text {AnPGI. }}$. Similarly, topologically equivalent residues in AnPGII and FmPG were identified that could impede such binding; $\mathrm{S} 234_{A n P G I} / \mathrm{S} 91_{\text {AnPGII }}$ and S245 $5_{F m P G} / V 89_{F m P G}$. In addition, we report here, from normal mode analysis computed on AnPG1, a shear bending motion of $15 \AA$ of amplitude that fully accredits the processive action pattern for this enzyme, with D240 ${ }_{A n \text { PGI }}$ and $\mathrm{R} 96_{\text {AnPGI }}$ working as crampons to favour the sliding of the substrate. Conversely, the same method clearly evidences a hinge binding motion for AnPGI and FmPG that should only authorize one hydrolytic event per enzyme/substrate encounter.
\end{abstract}

(c) 2008 Elsevier B.V. All rights reserved.

\section{Introduction}

Pectins are among the most abundant polysaccharides in many plant primary cell walls. Together with hemicelluloses, they define the two major networks in which the cellulose microfibrills are embedded. Pectin molecules are composed of different structural regions, which have been extensively described in previous reviews [1-3]. The homogalacturonan regions (HG) consist of a repetition of $\alpha$ (1, 4)-linked D-galacturonic acid (GalA). The rhamnogalacturonan regions $I(R G I)$ are constituted by an alternating sequence of $\alpha(1,4)$ linked D-galacturonic acid and $\alpha(1,2)$-linked rhamnopyranosyl residues. Various side chains (mainly arabinan, galactan or arabinogalactan) can link those rhamnose residues. The nature and lengths of the side chains vary among plant species. GalA in pectin can be partially methyl and/or acetyl-esterified. The degree of substitution

\footnotetext{
* Corresponding author.

E-mail address: bonnin@nantes.inra.fr (E. Bonnin).
}

(including acetylation) is defined as the number of substituents (acetyl groups) for each $100 \mathrm{GalA}$. A minor type of rhamnogalacturonan (RGII) shows a very complex structure composed of 12 different monomers. The structures of the HG and RG regions are quite well known but their way of combination remains unclear [4].

Basically, homogalacturonan is the best substrate for endopolygalacturonases (poly [1, 4- $\alpha$-D-galacturonide] glycano-hydrolase, EC 3.2.1.15, endoPGs) as endoPGs hydrolyse $\alpha(1,4)$ linkages between two GalA residues [5]. They are widely distributed in plants, fungi, yeasts and bacteria [6] and are the subject of a thorough research including their purification, properties and mode of action [for reviews see $[7,8]]$. Based on their sequences and their structurally related catalytic folding, endoPGs have been classified in glycoside hydrolase family 28 , GH28 [9] (http://afmb.cnrs-mrs.fr/CAZY/). It has been shown that family 28 enzymes cleave glycosidic bonds with inversion of the anomeric configuration [10]. Their activity is highly modulated by the presence of non-sugar substituents such as methyl and acetyl groups [11-13]. In a previous paper, we investigated the action pattern of 
Fusarium moniliforme PG (FmPG) towards poorly to highly methylated pectin fragments using molecular modelling and bioinformatics [14]. The tolerance of the enzyme towards methyl groups was understood in comparison with AnPGII known to be strictly non-tolerant to methyl-esters [12]. Amino acids that could have an impact on the behaviour of each enzyme towards defined methylated substrates were identified. Afterwards, the study was extended to acetyl-esters. An extensive degradation of highly acetylated pectin from sugar beet was carried out by AnPGI, AnPGII and FmPG and their hydrolysis products were carefully analyzed by mass spectrometry [15]. From that study, the most depictive products were selectively docked into each of the PG grooves. Our docking energies all showed accurate correlation with the hydrolytic schemes of the three PGs. In addition to the molecular docking, the electrostatic signature as well as the intrinsic flexibility through normal mode analysis was computed and assessed for each PG. Those molecular features clearly impact the tolerance of AnPGI, AnPGII and FmPG towards acetyl groups and amino acids that could guarantee or impede the processive hydrolysis of the PGs have been identified.

\section{Materials and methods}

\subsection{Coordinates of the enzymes}

We used the atomic coordinates of the native AnPGI, AnPGII and FmPG proteins that were solved at 1.70,1.68 and $1.73 \AA$ And deposited in the PDB bank under accession number 1nhc, 1czf and 1hg8, respectively (http://www.rcsb.org/pdb/home/home.do) [16-18]. They share the same single stranded right-handed $\beta$-helix topology, the same catalytic set of aspartate residues D191 ${ }_{F m P G}$, D212 $2_{F m P G}$ and D213 $F m$ PG (FmPG numbering) and thus the same inverting catalytic mechanism.

\subsection{Sequence alignment}

The sequences of AnPGI, AnPGII and FmPG were retrieved from the PDB databank in the PDB format (http://www.rcsb.org/pdb/home/ home.do), whereas the precursors of the proteins were extracted from the Uniprot database with P26213, P26214 and Q07181 accession numbers, respectively. Multiple alignments were performed with the ClustalW algorithm with standard parameters.

\subsection{Construction of the substrates}

The following abbreviations will be used to describe the oligomers produced by the enzymes: $G$ is the shortcut of GalA, $M$ the one of methylated GalA, $\mathrm{A}_{2}$ the abbreviation of GalA acetylated on $0-2$ and finally $A_{3}$, the abbreviation of GalA acetylated on $0-3$. For instance, $\mathrm{A}_{3} \mathrm{MG}$ is a trimer in which the non reducing end is acetylated on $0-3$, the central residue is methylated and the reducing end is free of substituent. In addition, there is no way to discriminate from which side of the product, the cleavage occurred. For instance, the fraction $\mathrm{GA}_{2}$ could either come from the hydrolysis of $\mathrm{GG} / \mathrm{GA}_{2}$ or $\mathrm{GA} / \mathrm{GG}$, so the two dockings shall be considered. The decorated oligomers were built on the parental scaffold of the linear GalA oligomer and positioned with respect to their position in the complex with FmPG [14]. To position the substrates into AnPGI and AnPGII crevices, the two PGs were successively superimposed onto the complex FmPG/ oligoGalA, using the pairwise structure tool from the ebi website (http://www.ebi.ac.uk/DaliLite/) [19].

\subsection{Molecular modelling}

\subsubsection{Electrostatic profile}

The electrostatic profile was calculated for each of the three PGs using the APBS (Adaptive Poisson-Boltzmann Solver) tool interfaced in Pymol (http://www.pymol.org) [20,21] after a PDB2PQR conversion [22] using most of the default parameters except the $\mathrm{pH}$ that was set up at 4 , where the degradations were carried out [15]. The former program evaluates the electrostatic properties of nanoscale biomolecular systems using the Poisson-Boltzmann equation (PBE), which is one of the most popular continuum models for describing electrostatic interactions between molecular solutes in salty, aqueous media. The latter program includes several steps as it first adds a limited number of missing heavy atoms to biomolecular structures, determines sidechain pKas, places missing hydrogens, optimizes the protein for favourable hydrogen bonding and finally assigns charge and radius parameters for a given forcefield; here AMBER was chosen.

\subsubsection{Normal mode analysis}

Normal mode analysis is a classical technique to generate functionally relevant movements in biological macromolecules. The principle is to use an all atom model with a highly simplified and quadratic potential energy between atoms that are considered to be linked by a spring of universal strength provided that they are located less than $10 \AA$ away in the PDB structure [23]. For low-frequency normal modes, which give large-amplitude and collective modes, this Elastic Network Model gives excellent results. We used the corresponding NOMAD program to compute the flexibility of each PG into motion modes of low frequency (http://lorentz.immstr.pasteur.fr/ nomad-ref.php) [24]. With the PDB coordinates of the PGs as input files, NOMAD calculates and explores each motion shaping that associates large-amplitude movements and correct stereochemistry.

\subsubsection{Docking of substrates}

Molecular docking was carried out on an SGI computer with the Accelrys $₫$ package (Accelrys. Inc, San Diego, CA, USA). Molecular displays and energy minimisations were performed using the molecular and simulation modelling environment InsightII, combining Biopolymer and Discover modules. When necessary, tools of the recently transferred InsightII version of linux Discovery Studio@ have been used. To compare our data with our previous work on FmPG complexed to methylated pectin, similar cycles of docking and optimization were used. The strategy implies the CFF91 force field and steepest descent minimization algorithm during which the backbone of the protein was kept fixed and the side chains and sugar moieties allowed to relax (15,000 iterations) [14,25,26]. We computed similar energetic criteria as previously defined: 1 . potential energy of the protein, 2. potential energy of the substrate, 3. potential energy of the complex and 4. binding energy of the complex, which is exclusively the contribution of the residues involved through van der Waals and electrostatic interactions with the oligosaccharide. The binding energy can be directly correlated to the stability.

\section{Results}

\subsection{Docking of oligomers in the catalytic groove}

From our previous experimental study, GalA trimer, tetramer and pentamer were found to be the archetypal products resulting from pectin hydrolysis by the three enzymes [15]. They are all methyl- and/ or acetyl-esterified and released in appreciable quantities whatever the enzyme used. $A_{3} M G$ is the most predominant trimer produced. The corresponding substrate $A_{3} M G / G G$ was thus built for subsequent docking in the catalytic groove of each PG. Conversely, $\mathrm{DP}_{4}$ and $\mathrm{DP}_{5}$ are delivered with various substitution patterns where the most abundant configurations are $\mathrm{GA}_{2} \mathrm{MG}$ and $\mathrm{GA}_{2} \mathrm{MGG}$. Similarly, $\mathrm{GA}_{2} \mathrm{MG} / \mathrm{GG}$ and $\mathrm{GA}_{2} \mathrm{MGG} / \mathrm{GG}$ were built for further docking. The binding energies for all the modelled complexes are registered in Table 1. AnPGI evidences stronger binding, regardless of the substrate length, as compared to the two others. AnPGI shows the best binding energy for $\mathrm{GA}_{2} \mathrm{MG} / \mathrm{GG}$ with $-186 \mathrm{kcal} / \mathrm{mol}$, so when $\mathrm{GA}_{2} \mathrm{MG}$ spans from subsite -4 to -1 . This 
Table 1

Binding energies (in $\mathrm{kcal} / \mathrm{mol}$ ) for AnPGI, AnPGII and FmPG in complex with oligogalacturonates

\begin{tabular}{llll}
\hline Binding energy & AnPGI & AnPGII & FmPG \\
\hline $\mathrm{GA}_{2} / \mathrm{GG}$ & -135 & -112 & -115 \\
$\mathrm{GG}_{\mathrm{GA}}$ & -131 & -113 & -124 \\
$\mathrm{GA}_{3} / \mathrm{GG}$ & -114 & -94 & -119 \\
$\mathrm{GG} / \mathrm{GA}$ & -135 & -106 & -108 \\
$\mathrm{~A}_{3} \mathrm{MG} / \mathrm{GG}$ & -141 & -113 & -140 \\
$\mathrm{GA}_{2} \mathrm{MG} / \mathrm{GG}$ & $\mathbf{- 1 8 6}$ & -129 & -159 \\
$\mathrm{GA}_{2} \mathrm{MGG} / \mathrm{GG}$ & -165 & $\mathbf{- 1 5 0}$ & $\mathbf{- 1 7 6}$ \\
\hline
\end{tabular}

The most stable complex for each enzyme, suggested by the lowest energy, is highlighted in bold.

is fully relevant with the product quantification [15], where $\mathrm{GA}_{2} \mathrm{MG}$ was measured as the oligomer released in the highest quantity by AnPGI action. AnPGII and FmPG both display much lower binding energies for those short substrates. Thus, the three enzymes show a binding profile that correlates very well with the experimental hydrolytic pattern. Particularly, the percentage of products with a degree of polymerisation lower than 6 was markedly lower for AnPGII and FmPG, 64\% and 56\% of total GalA respectively as compared to 75\% for AnPGI. In addition, the docking study shows that AnPGII and FmPG, with binding energies of -150 and $-176 \mathrm{kcal} / \mathrm{mol}$ respectively, give more stable complex with the fraction $\mathrm{GA}_{2} \mathrm{MGG} / \mathrm{GG}$. This is fully consistent with the experimental data that assessed this moiety as the most abundant oligomer produced by those enzymes [15].

Our docking study evidences that AnPGI gives more stable complexes for oligosaccharides with $A_{2}$ and $A_{3}$ acetylations as compared to the two other PGs. This is again in full accordance with the experimental data that assessed AnPGI as the most potent enzyme and the most tolerant towards acetylated fragments [15]. In more details, Table 1 shows that the three enzymes display a markedly higher binding energy for $A_{2}$ acetylation as compared to $A_{3}$, at subsite -3. For instance, for AnPGII, $-129 \mathrm{kcal} / \mathrm{mol}$ for $\mathrm{GA}_{2} \mathrm{MG} / \mathrm{GG}$ has to be compared to $-113 \mathrm{kcal} / \mathrm{mol}$ for $A_{3} M G / G G$, and the difference is in the same order whatever the enzyme. Similarly at the subsite $-1, A_{2}-$ is better tolerated than $A_{3}$ - acetylation, as for instance, $-135 \mathrm{kcal} / \mathrm{mol}$ for $\mathrm{GA}_{2} / \mathrm{GG}$ compared to $-114 \mathrm{kcal} / \mathrm{mol}$ for $\mathrm{GA}_{3} / \mathrm{GG}$ in the case of acetylated GalA in subsite -1 of AnPGI. This is consistent with higher amount of $\mathrm{A}_{2}$ oligosaccharides compared to $\mathrm{A}_{3}$ ones that were quantified after enzymatic digestion [15]. The only exception is for subsite -1 in FmPG that shows rather equal binding affinity between $\mathrm{GA}_{2} / \mathrm{GG}$ and $\mathrm{GA}_{3} / \mathrm{GG}$ with $-119 \mathrm{kcal} / \mathrm{mol}$ and $-114 \mathrm{kcal} / \mathrm{mol}$, respectively. In any case, it is consistent with the tolerance profile of the three PGs that all accept $A_{2}$ and $A_{3}$ in subsite -1 .

\subsection{Comparison of the three proteins}

GH28 family enzymes are conformed as single stranded righthanded beta-helix fold. The catalytic triad D191 ${ }_{\mathrm{FmPG}}, \mathrm{D} 212_{\mathrm{FmPG}}$, D213 ${ }_{F m P G}$, plus H188 ${ }_{F m P G}, N 189_{F m P G}$, T190 ${ }_{F m P G}, H 234_{F m P G}, \mathrm{R}^{267_{F m P G}}$ and $\mathrm{K} 269_{\mathrm{FmPG}}$ are strictly conserved among the overall family (see alignment of AnPGI, AnPGII and FmPG on Fig. 1) [16-18]. These conserved residues are involved in the subsites -1 and +1 having the highest affinity for GalA [14]. Sequence analysis of the three enzymes, through 1D alignment, evidences a high homology degree with 30\% strict identity shared. As expected and already published [16], AnPGI and AnPGII share 57\% strict identity. FmPG sequence is slightly more similar to AnPGII sequence than to AnPGI with aligned sequence score of $43 \%$ and $40 \%$, respectively. The pairwise structure comparison by $3 \mathrm{D}$ alignment using AnPGI as the reference shows an average rms

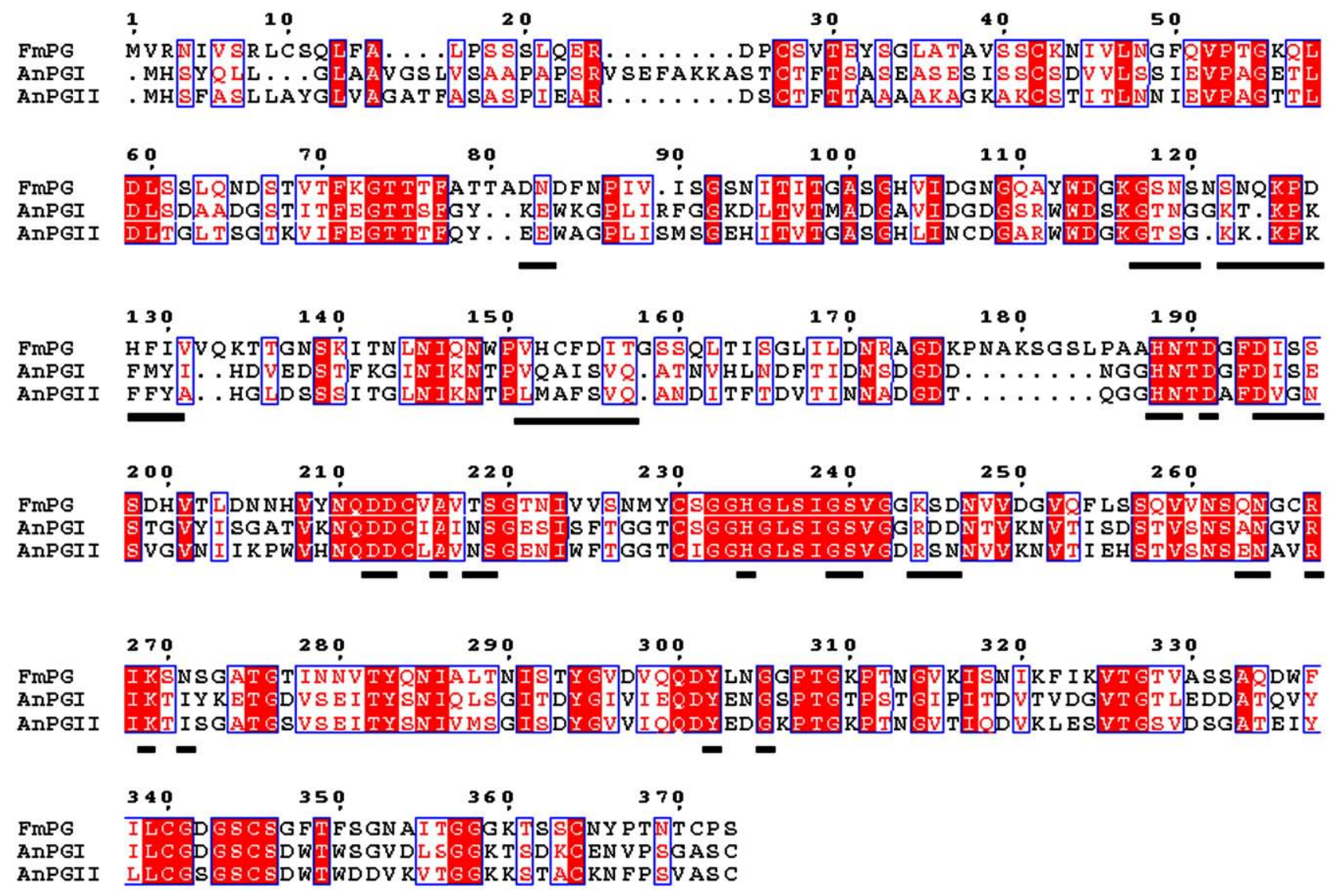

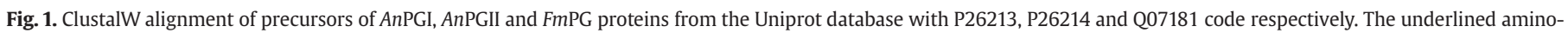
acids belong to the catalytic groove of at least one of the three enzymes. 
deviation of $0.8 \AA$ and $1.5 \AA$ A between equivalent $\mathrm{C} \alpha$ for AnPGII and FmPG, respectively. Basically, the residues that cover the catalytic cavity are very well conserved among the three PGs. Still, some discrepancies on the sequences can be pinpointed as they are visible on the clefts and could be crucial for substrate tolerance or processivity pattern. The regions of the 1D sequence covering the catalytic groove are underlined on Fig. 1 below the alignment. Some notable differences in or next to the catalytic clefts are one amino-acid insertion $\mathrm{N} 83_{\mathrm{FmPG}}$, one amino acid insertion $\mathrm{R} 6_{\text {AnPGI }} / \mathrm{S} 91_{\text {AnPGII }}$ that $3 \mathrm{D}$ aligned with $\mathrm{V} 9_{\mathrm{FmPG}}$, a motif of low complexity $\mathrm{S} 118_{\mathrm{FmPG}} \mathrm{NSN} 123_{\mathrm{FmPG}}$,

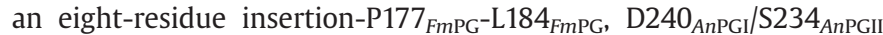
that $3 \mathrm{D}$ aligned with $\mathrm{S} 245_{\mathrm{FmPG}}$ and eventually the variation region
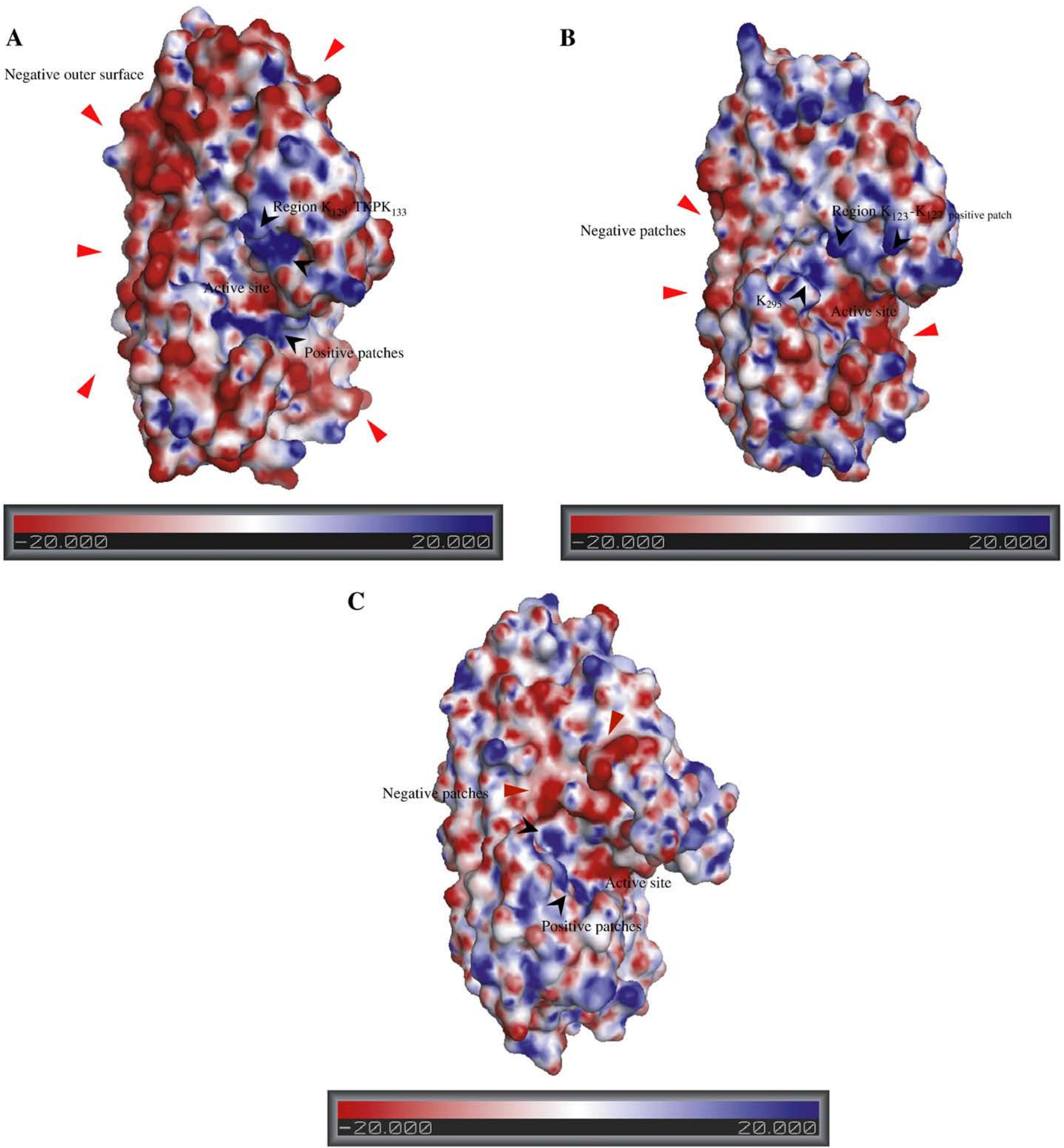

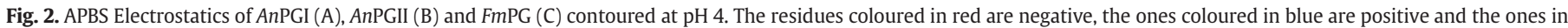

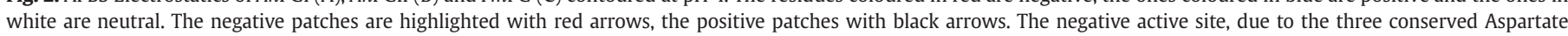
residues, is mentioned. The electrostatics has been homogeneously scaled between -20 and +20 for each. 
L303 ${ }_{F m P G}-G 306_{F m P G}$. Only V89 $9_{F m P G}$ and residues equivalent in AnPGs $\left(\mathrm{R} 6_{\text {AnPGI }} / \mathrm{S} 1_{\text {AnPGII }}\right)$ belong to a structural $\beta$ strand, the other residues belong to loops or to T3 turns.

The calculation of the electrostatic surface clearly shows distinct profiles for the three PGs (Fig. 2A-C). At pH 4, where the degradation was carried out [15], AnPGI, AnPGII and FmPG have a total charge of $-6,+9$ and +5 , respectively. The distribution of charge in AnPGI evidences an outer surface highly negative combined to a catalytic groove that contains the positive lysine rich motif K129 AnPGI $_{\text {TKPK133- }}$ AnPGI, where $\mathrm{K} 133_{\mathrm{AnPG}}$ is in position to interact with the substrate at subsites $-2 /-1$, consequently highly attractive to a negative demethylated pectate (Fig. 2A). AnPGII shows an outer surface less negative and a catalytic crevice slightly more negative as compared to AnPGI. However, the enzyme associates positive patches at the apex of two loops that face each other apart from the fissure, on one side the lysine

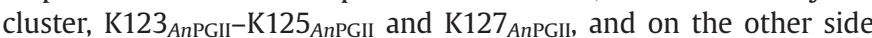
K295 (Fig. 2B). Finally, the electrostatic footprint is less pronounced for FmPG that displays a balanced and scattered positive/negative outer surface and the inner fissure the less charged among the three (Fig. 2C).The docking study maps the subsites in AnPGI and AnPGII and evidences that both enzymes have a 7 subsites active site that split into five $(-5$ to -1$)$ and two $(+1$ to +2$)$ apart from the catalytic site that spans subsites -1 and +1 . Similarly, FmPG active site defines up to 7 subsites that cover from -5 to +2 , thus confirming an additional subsite when compared to our previous results [14]. From the computational docking, we were able to list precisely the amino acids involved in the binding of oligogalacturonates through apolar, polar and electrostatic contributions (Table 2). Interestingly, remarkable differences in the electrostatic signature of each PG can be zoomed in at subsites $-4,-5$ and +2 for the three enzymes, the inner subsites being most homogeneous. AnPGI clearly shows a negatively

Table 2

List of amino acids of AnPGI, AnPGII and FmPG involved, either through hydrogen bonds, van der Waals interactions or salt bridges, in the binding of decorated oligogalacturonates

\begin{tabular}{|c|c|c|c|}
\hline & AnPGI & AnPGII & FmPG \\
\hline \multirow[t]{3}{*}{ Subsite - 5} & Q161 & Q155 & T157 \\
\hline & E192 & N186 & S197 \\
\hline & D240 & S234 & S245 \\
\hline \multirow[t]{4}{*}{ Subsite - 4} & Q161 & Q155 & T157 \\
\hline & E192 & N186 & S197 \\
\hline & D240 & S234 & S245 \\
\hline & D241 & N235 & D246 \\
\hline \multirow[t]{6}{*}{ Subsite - 3} & K133 & K127 & $\mathrm{H} 128$ \\
\hline & S159 & S153 & D155 \\
\hline & V160 & V154 & I156 \\
\hline & S191 & V184 & S196 \\
\hline & N213 & S208 & S219 \\
\hline & D240 & S234 & S245 \\
\hline \multirow[t]{5}{*}{ Subsite - 2} & D189 & D183 & D194 \\
\hline & I190 & V184 & I195 \\
\hline & A211 & A205 & A216 \\
\hline & N213 & N207 & $\mathrm{T} 218$ \\
\hline & R239 & R233 & K244 \\
\hline \multirow[t]{7}{*}{ Subsite - 1} & T125 & $\mathrm{T} 120$ & S118 \\
\hline & V155 & L149 & V151 \\
\hline & Q156 & M150 & H152 \\
\hline & D186 & D180 & D191 \\
\hline & G234 & G228 & G239 \\
\hline & S235 & S229 & S240 \\
\hline & K264 & K258 & K269 \\
\hline \multirow[t]{6}{*}{ Subsite + 1} & N126 & $\mathrm{S} 121$ & N119 \\
\hline & N184 & N178 & N189 \\
\hline & D207 & D201 & D212 \\
\hline & D208 & D202 & D213 \\
\hline & Y297 & Y291 & Y302 \\
\hline & S301 & K295 & G305 \\
\hline
\end{tabular}

The catalytic residues are in italic. Amino acids with non polar side chains are in black, amino acids with uncharged polar side chains are in green, amino acids with positively and negatively charged polar side chains are in blue and red, respectively. Amino acids in bold are proposed for site directed mutation. charged surface at subsites -4 and -5 with E192, D240 and D241 and, reversely, a highly positively charged surface at subsite +2 with $\mathrm{H} 183$, H229 and R262 (Fig. 3A). AnPGII displays a highly polar surface at subsites -4 and -5 with residues Q155, N186, S234 and N235 and a polar charged at subsite +2 with H177, H223, E252 and R256 (Fig. 3B). Finally, FmPG stands in between the two other profiles with a highly polar surface at subsites -4 and -5 with residues T157, S197, S245 and S246 and a highly positively charged surface at subsite +2 with residues H188, H234 and H267 (Fig. 3C).

\subsection{Processivity pattern through NOMAD analysis}

The default parameters of NOMAD compute 16 motion modes. The first six are discarded as they refer to the own translations and rotations of the protein. The first motion mode that can be relevant starts at 7 . Indeed, the motion mode analysis evidences remarkable flexibility at modes 7 and 8 that are shear bending and hinge bending motions for the three PGs. Shear bending flexibility is characterized by the glissade of the $\mathrm{N}$-terminus portion along the $\mathrm{C}$-terminus one, apart from the catalytic cleft (See arrows in Fig. 4A). The hinge bending motion describes the successive opening or closure of the same domains over the active groove (See arrows in Fig. 4B, C). Interestingly, despite a strictly conserved topology and a high sequence identity, the differences in scaling motions between hinge and shear bending are outstanding between AnPGI on one hand and AnPGII and FmPG on the other hand. Clearly, AnPGI promotes a straightforward shear bending motion with measured amplitude of 15 A between the $\mathrm{C} \alpha$ of $\mathrm{N}^{126} 6_{\text {AnPGI }}$ and the carbonyl oxygen of $\mathrm{N}_{29} 9_{\mathrm{AnPGI}}$. Those residues define the two extreme positions along the trajectory (Fig. 4A). Indeed, N126 $6_{\text {AnPGI }}$ and N299 ${ }_{\text {AnPGI }}$ are located at the apex of loops apart from the catalytic site and submitted to the most substantial amplitude of motions. The distance variations between the two residues, measured during hinge bending motion exhibits a rather weak amplitude of $5 \AA$ in AnPGI. Reversely, AnPGII and FmPG definitely favour the opening/closure flexibility of the hinge bending motion as shown by the amplitudes of $24 \AA$ and $18 \AA$, respectively, measured between $\mathrm{C} \alpha$ and carbonyl oxygen of the homologous residues S121 ${ }_{\text {AnPGII }}$, D293 ${ }_{\text {AnPGII }}$ and $\mathrm{N}_{118}{ }_{\mathrm{FmPG}}, \mathrm{N} 304_{\mathrm{FmPG}}$ (Fig. 4B, C). It is noteworthy that the shear bending motion is weaker for AnPGII and FmPG with amplitudes of $4.5 \AA$ and $9 \AA$, respectively.

\section{Discussion}

From previous experimental data and our in silico calculations, it is clear that the three PGs, despite sharing a common architecture, a similar subsite distribution with a strictly conserved catalytic mechanism, diverge in substrate binding specificity, in tolerance behaviour towards acetylated or methylated pectins and in processivity pattern. We advance here molecular clues that should explain those macromolecular properties and we propose site-directed mutations to validate our assumptions.

It was previously demonstrated that acetyl groups $0-2$ or $0-3$ on homogalacturonan GalA backbone are energetically favourable and that the presence of acetyl groups did not alter the conformational behaviour of the backbones [28]. It can also be calculated from the length of $\mathrm{C}-\mathrm{O}$ bond $(1.43 \AA$ ) and $\mathrm{C}-\mathrm{H}$ bond $(1.07 \AA)$ that the presence of an acetyl group induces a limited protuberance of around $18 \AA^{3}$. Compared to the volume of the active site cavity ( $849 \AA^{3}$ on average), it can be assumed that acetyl groups on the homogalacturonan backbone do not modify very much the geometry and the volume of the substrate and instead could favour the binding through hydrogen bonding. Indeed, we report for AnPGI possible hydrogen bonding between H138, S159, S191, E192 and R239 and acetyl group when positioned as $A_{2}$, or between Q161 and R239 and acetyl groups when positioned as $A_{3}$. Similarly for AnPGII, we list possible hydrogen bonding between R233 and N207 and acetyl group $A_{2}$. No hydrogen 
A
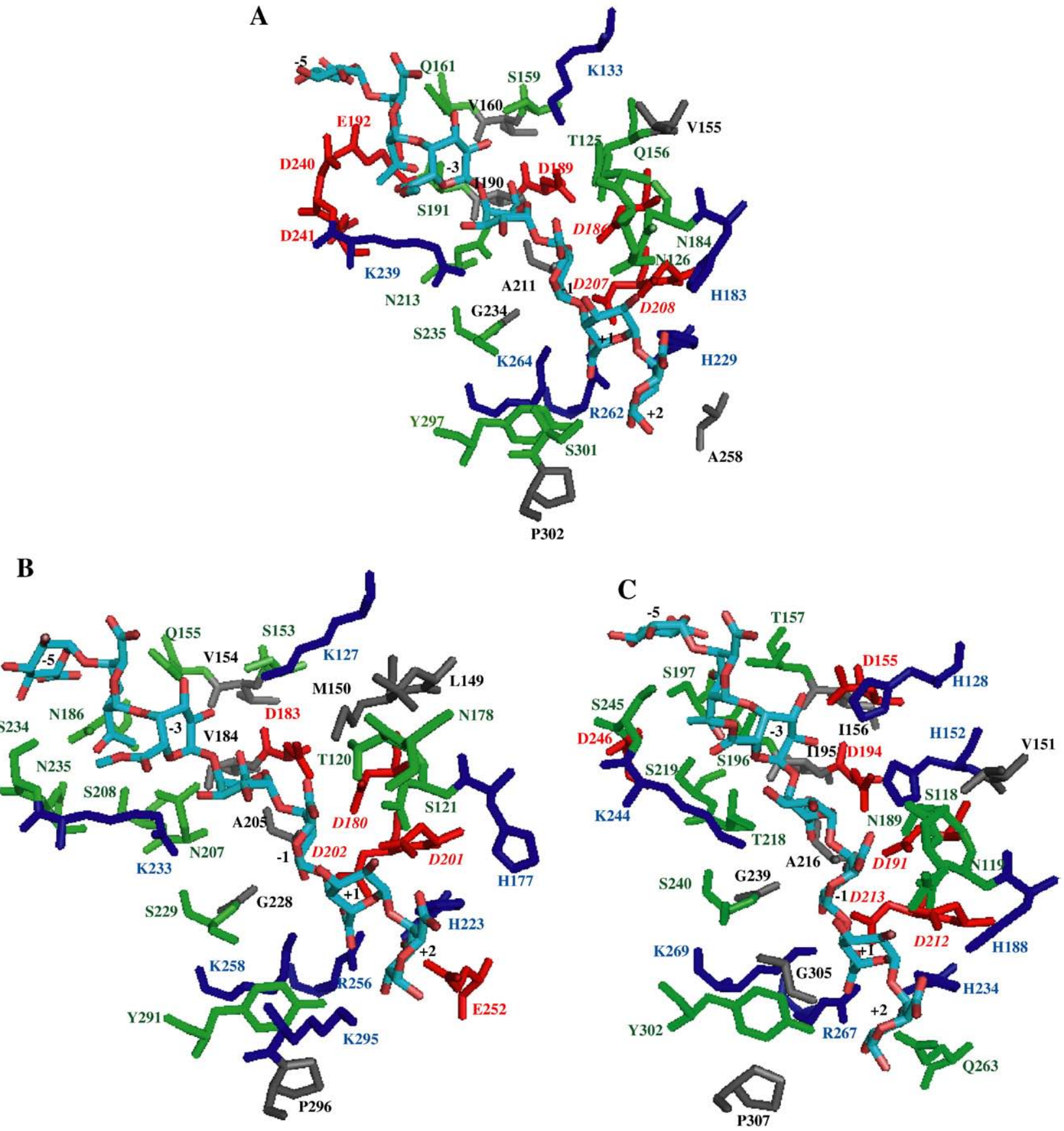

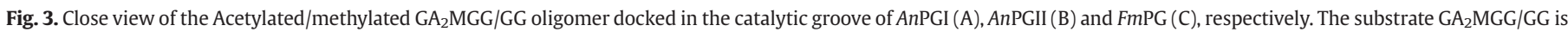

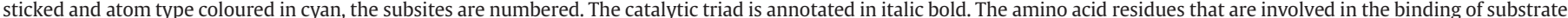

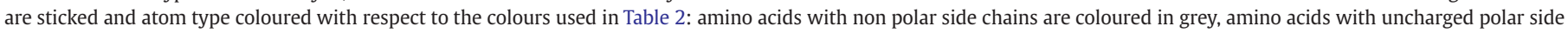
chains are in green, amino acids with positive charge are blue and with negative charge are red.

bonding can be listed between AnPGII and $\mathrm{A}_{3}$ or between FmPG and $\mathrm{A}_{2}$ or $\mathrm{A}_{3}$. Our molecular modelling study reveals binding profiles that associates very well with the experimental hydrolytic pattern of the three PGs. Indeed, AnPGI always evidences higher binding energies, as compared to AnPGII and FmPG, showing that AnPGI binds more strongly the oligogalacturonates, especially short ones. It is fully relevant as AnPGI is also the most efficient enzyme among the three PGs [15]. This is particularly true for $\mathrm{GA}_{2} \mathrm{MG} / \mathrm{GG}$, which displays the best energy when docked in AnPGI active site and from which $\mathrm{GA}_{2} \mathrm{MG}$ was the main product with $\mathrm{DP}_{4}$ [15]. AnPGII and FmPG both display much weaker binding energies for short substrates. This is in line with the experimental data that quantified the total of digested Gal A as markedly lower for the two enzymes as compared to AnPGI. In addition, the docking study shows that the highest binding energy is obtained for $\mathrm{GA}_{2} \mathrm{MGG} / \mathrm{GG}$ which is shown experimentally to be also the most abundant oligomer produced by those enzymes [15]. Our study evidences that the three PGs share a similar unequal distribution of subsites apart from the active subsites -1 and +1 with a rather conserved core of residues from subsites -2 to +1 . In line with that, we calculate that each PG has a completely different total charge for an identical pH of optimum activity, that the electrostatic contour is distributed contrastingly for the three enzymes, with interesting variations especially at the external subsites. We suggest that those distinct electrostatic properties could impact the binding. AnPGI 

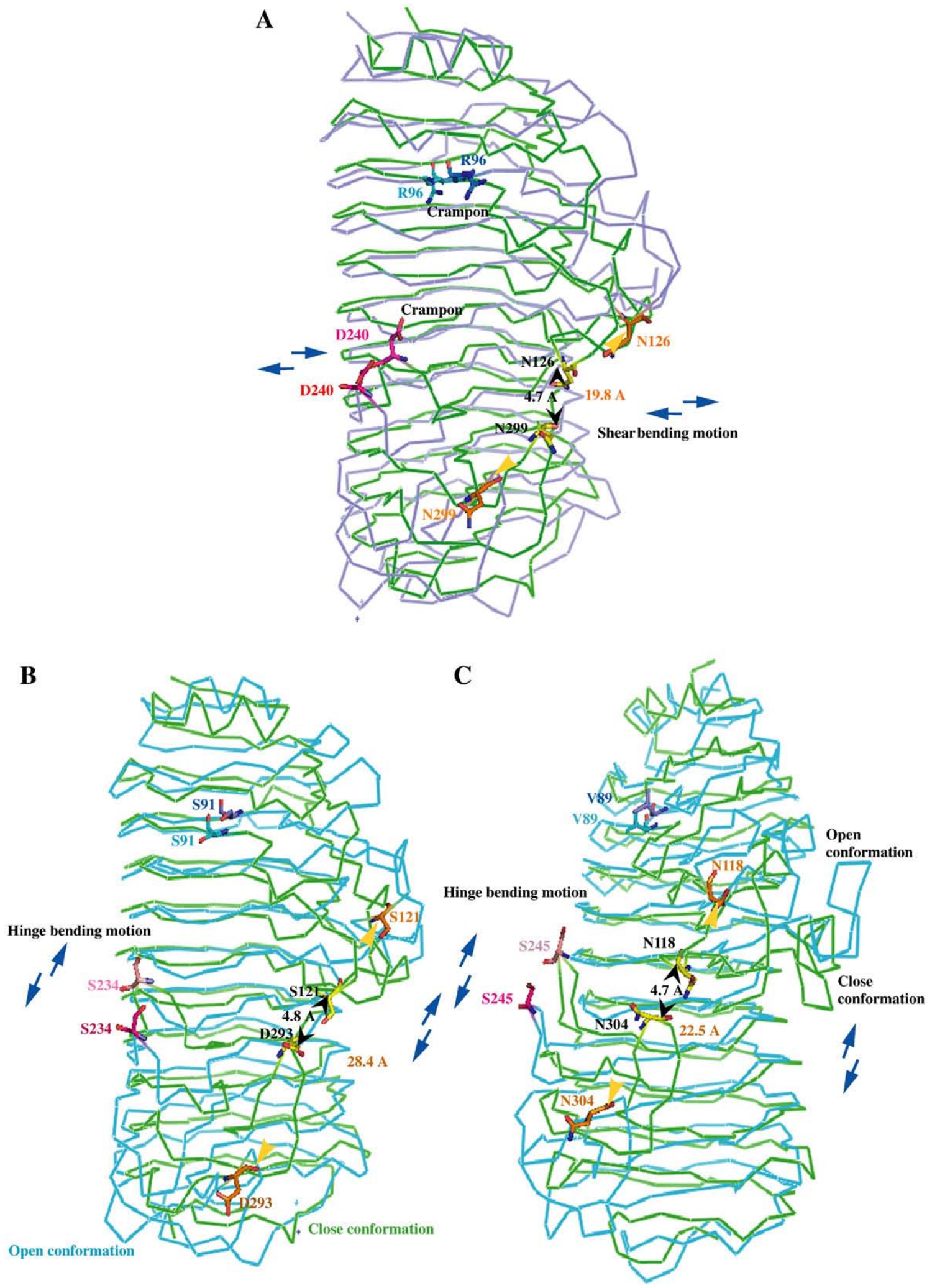

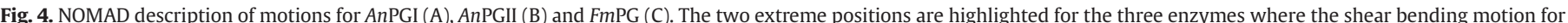

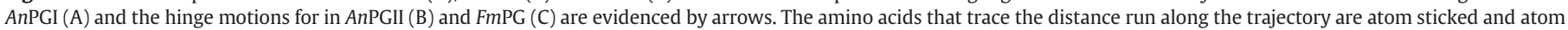

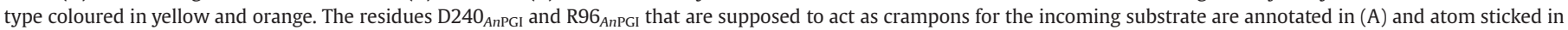
clear and deep pink. The equivalent residues S234 ${ }_{A n P G I}, S 91_{A n P G I}$ and S245 $5_{F m \text { PG }}$ V8 $9_{F m P G}$ are positioned in (B) and (C) for comparison.

displays a highly negative contour of surface with fissure subsites -5 and -4 clearly negative and subsite +2 markedly positive. The fact that AnPGI is far more potent could be due to a particularly efficient step of substrate binding as the negative outer surface could repel the negative pectate and drive it straightforwardly to the contrasting positive groove. AnPGII is the most positively charged, FmPG having 
an intermediate positive charge. Our results for AnPGI and AnPGII are in line with the very low tolerance of AnPGI and AnPGII towards methylated pectin, as the enzymes are clearly not active on this substrate [12]. On the opposite, our result for FmPG is in line with its relative tolerance for less charged methylated pectin [27]. In details, subsites -5 and -4 in AnPGII and FmPG are highly polar as exclusively composed of Asparagine, Serine, Threonine and Glutamine residues. Subsite +2 of AnPGII is less positive than subsite +2 of its counterpart AnPGI, this of FmPG is slightly more polar. Those elements together suggest that the polar patch at subsites -5 and -4 of AnPGII or FmPG could attract the substrate and be the key to the binding of rather long pectate substrate for which affinity to the protein seems to increase with the DP.

From our recent experimental work [15] and despite a narrower catalytic groove that one could expect to hamper the docking of decorated substrates, AnPGI is the most potent enzyme and the most tolerant one towards acetylated $\mathrm{A}_{2}$ and $\mathrm{A}_{3}$ pectate. FmPG is the most tolerant one to methylated pectins [13,14] and AnPGII is both less tolerant to $A_{2}, A_{3}$ pectate and to methylated pectins [15]. From the global electrostatic profile of each PG and the previous extensive study of methylated and not methylated substrates that were docked in the active site of FmPG [14], we gained molecular clues about the methylated substrate binding capacity [14] or acetylated substrate binding capacity. Methyl decorations abolish the negative charge of the pectate, rendering it more hydrophobic, more neutral and thus less sensitive to the electrostatic field contouring the protein. $A_{2}$ or $A_{3}$ are decorations that replace the hydroxyl groups on carbon $C_{2}$ and $C_{3}$, respectively. They do not screen any negative charge of the GalA moieties and thus do not change the sensitivity of the substrate towards positive or negative charges of the proteins. In that sense, AnPGI offers the most contrasting negative outer surface vs positive groove, so the latter can easily be perceived as highly attractive to a negatively charged pectate. FmPG is the one with the less pronounced electrostatic signature and thus the one most likely to accept the pectin substrate. If we zoom into the active cleft of each PG, we have identified a residue as possibly crucial to modulate such tolerance. Namely, due to its position on a protruding loop, D240 ${ }_{\text {AnPGI }}, S 234_{\text {AnPGII }}$ and $5245_{\text {FmPG }}$ define subsites $-3,-4$ and -5 in AnPGI, AnPGII and FmPG, respectively. Facing the residue and located at the end of a $\beta$ sheet on the other side of the cleft, we have $\mathrm{S} 191_{A n \mathrm{PGI}}, \mathrm{G} 185_{\text {AnPGII }}$ and

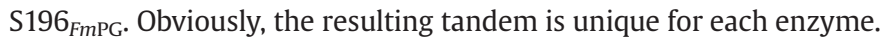
D240 ${ }_{A n P G I}$ has a long and charged side chain that is suspected to be responsible for the peculiar positioning of the substrate on the glycone side, as compared to the two others and $\mathrm{S} 191_{\text {AnPGI }}$ hydrogen bonds the $\mathrm{A}_{2}$ group in subsite -4 (Fig. $3 \mathrm{~A}$ ). $\mathrm{S} 234_{\text {AnPGII }}$ and $\mathrm{S} 245_{\text {FmPG }}$ could be responsible for a comparable global positioning of the substrate that the two enzymes share. However, S196 ${ }_{F m P G}$ hydrogen bonds the $A_{2}$ group in subsite -4 whereas $G 185_{\text {AnPGII }}$ does not, so we suggest that this hydrogen bond is critical to boost the tolerance (Fig. 3B, C). We propose $S 196_{F m P G}$ for mutation to gain some tolerance or D240 ${ }_{A n P G I} S$ reversely to decrease it.

The NOMAD motion modes explore the conformational space where we suggest that each PG could be subjected to allosteric conformational changes upon binding of substrate. AnPGI likely shows an exceptional shear bending motion of $15 \AA$ of amplitude that combines with a 5 Å weak opening. Reversely, AnPGII and FmPG possibly display hinge bending motion of 24 and $18 \AA$, respectively, that combines with a weaker shear bending motion of 4.5 and $9 \AA$, respectively. These motions should not only enable the correct positioning of the oligogalacturonates in the active site but should favour or impair the processive pattern of each enzyme. In AnPGI, the exceptional shear flexibility combined with a weak opening suggests that the substrate could slide along the crevice after its cleavage with only partial unbounding, for an ensuing hydrolysis. The narrow cleft of the enzyme topologically supports this hypothesis. Indeed, processive enzymes attack the same polymer chain repeatedly during one encounter and in a narrow cleft, the entrapped substrate is more likely to slide along the subsites than to be released. This is actually fully relevant with the work of van Pouderoyen and coworkers who assessed experimentally that AnPGI excised processively several GalA residues of pectate at one enzyme-glycan encounter [16]. Reversely, we suggest that the groove opening, clearly observed for AnPGII and FmPG, may be required upon catalysis to release the substrate once cleaved. This is in line with the experimental results that clearly evidence a non-processive scheme for those two enzymes [14,16,19].

In details, $\mathrm{R} 6_{A n \mathrm{PGI}}$ has been reported as crucial for the processivity of AnPGI [16]. The authors have abolished the positive charge of R96 $6_{A n \mathrm{PGI}}$ (located behind subsite -4 ) with a mutation into a Serine residue and consequently the enzyme is not longer processive. Reversely, when AnPGII that possesses S91 ${ }_{A n \text { PGII }}$ at this position is mutated into an Arginine residue, the enzyme acquires processivity. They suggested that the positive charge of the Arginine residue binds more strongly the negatively charged pectin than the Serine, thus emphasizing the glissade of the substrate along the active site. In line with this, we observed that, along AnPGI trajectory motion, this residue comes to face the residue $\mathrm{D} 240_{A n P G l}$, positioned on the Cterminal side and identified as part of subsites -4 and -5 . Those two residues with long side chain and opposite charge apart from the crevice could act as crampons onto the substrate to help the sliding and favour the processivity. As was already mentioned earlier in this paper, AnPGII possesses S234 $4_{\text {AnPGII }}$ at position equivalent to $\mathrm{D} 240_{\text {AnPGI }}$. This residue has a non charged shorter side chain that could not compensate D240 ${ }_{A n \mathrm{PGI}}$ in acting as crampon for the substrate. FmPG neither displays an Arginine nor a Serine residue at position equivalent to $\mathrm{R} 96_{A n \mathrm{PGI}}$ or $\mathrm{S} 91_{A n \mathrm{PGII}}$. In $3 \mathrm{D}$ structure, this position is occupied by the short length and hydrophobic Valine residue V89 $9_{\text {FmPG. }}$. In position equivalent to D240 ${ }_{A n P G}$, FmPG has $\mathrm{S} 245_{\mathrm{FmPG}}$ and FmPG is

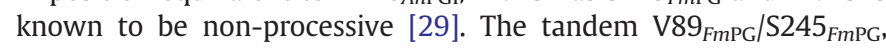
similarly to the couple $S 91_{\text {AnPGII }} / \mathrm{S} 234_{\text {AnPGII }}$ could be insufficient to clamp the substrate for another round of hydrolysis. We propose that V89 $9_{\text {Fm PG }}$ could be rationally substituted into an Arginine residue to test the conversion into a processive enzyme.

\section{Conclusion}

Sequence alignment of three highly homologous enzymes, AnPGI, AnPGII and FmPG, calculation of their electrostatic contour, and computation of their intrinsic flexibility have been extensively combined to the docking of poorly to highly decorated substrates to comprehend, at a molecular scale, their differences in the tolerance profiles towards acetylated pectins or in their processive-or notpattern of hydrolysis. Our work suggests that, besides the topology of the catalytic cleft that goes from narrow with AnPGI to large with AnPGII, motion flexibility should be pivotal for processivity property. Indeed, the appreciative shear bending motion of AnPGI should favour the processive pattern whilst the hinge binding motion observed in AnPGII and FmPG should conversely favour the release of substrate after one catalytic event. The tandem of residues, $\mathrm{D} 240_{\text {AnPGI }} / \mathrm{R} 6_{\text {AnPGI }}$, that is $1 \mathrm{D} / 3 \mathrm{D}$ replaced by $\mathrm{S} 234_{A n \mathrm{PGI}} / \mathrm{S} 91_{\mathrm{AnPGII}}$, and by $\mathrm{S} 245_{\mathrm{FmPG}} /$ $\mathrm{V} 89_{\mathrm{Fm} P \mathrm{PG}}$ is unique and has large-amplitude deviation during the identified motions. We suggest that the couple D240 ${ }_{\text {AnPGI }} / \mathrm{R} 96_{\text {AnPGI }}$ could dynamically come over the catalytic cleft to clamp the substrate. This could be pivotal to enhance the sliding of the substrate along the fissure. In addition, we suggest that the electrostatic contour outside of and inside the catalytic cleft explains how AnPGI is not only the most potent but also the most tolerant enzyme to hydrolyse acetylated pectin. We have identified the couple $S 191_{A n \mathrm{PGI}} / \mathrm{D} 240_{\mathrm{AnPGI}}$ as critical for the binding of acetylated substrate. Clearly from this work, D240 ${ }_{\text {AnPG }}$ has been identified as crucial for the processivity when in tandem with R96 $6_{A n P G I}$ but also for the tolerance profile of the enzyme towards acetylated substrate when this time in association to S191 $1_{A n \mathrm{PGl}}$. Combinations of site-directed substitutions according to 
the residue variations occurring in the other two should be performed experimentally to confirm our in silico procedure as powerful and successful in identifying PGs mutations that could modulate the tolerance as well as the hydrolysis pattern profiles.

\section{Acknowledgement}

The authors wish to thank Dr Marie-Christine Ralet for fruitful discussions about the structure of the produced oligomers.

\section{References}

[1] A.G.J. Voragen, W. Pilnik, J.-F. Thibault, M.A.V. Axelos, C.M.G.C. Renard, Pectins, in A.M. Stephen (Ed.), Food Polysaccharides and Their Applications, Marcel Dekker New-York, 1995, pp. 287-340.

[2] H. Schols, A.G.J. Voragen, Complex pectins: structure elucidation using enzymes, in: J. Visser, A.G.J. Voragen (Eds.), Pectins and Pectinases, Elsevier Science B.V. Amsterdam (The Netherlands), 1996, pp. 3-19.

[3] M.-C. Ralet, E. Bonnin, J.-F. Thibault, Pectins, in: A. Steinbüchel (Ed.), Biopolymers, vol. 8, Wiley-VCH Verlag Gmbh, Weinheim, 2003, pp. 345-380.

[4] J.-P. Vincken, H.A. Schols, R.J.F.J. Oomen, M.C. McCann, P. Ulvskov, A.G.J. Voragen, R.G.F. Visser, If homogalacturonan were a side chain of rhamnogalacturonan I. Implications for cell wall architecture, Plant Physiol. 132 (2003) 1781-1789.

[5] H. Neukom, Uber den Abbau von Pektinstoffen, Schweitz. Landwirtsch. Forsch 2 (1963) 112-122.

[6] W.M. Fogarty, C.T. Kelly, Pectic enzymes, Microbial Enzymes and Biotechnology, Applied Science Publishers, London (UK), 1983, pp. 131-182.

[7] T. Sakai, T. Sakamoto, J. Hallaert, E.J. Vandamme, Pectin, pectinase and protopectinase: production, properties and applications, Adv. Appl. Microbiol. 39 (1993) 213-294.

[8] C. Lang, H. Dönenburg, Perspectives in the biological function and the technological application of polygalacturonases, Appl. Microbiol. Biotechnol. 53 (2000) 366-375.

[9] B. Henrissat, G.J. Davies, Structural and sequence-based classification of glycoside hydrolases, Cur Opin. Struct. Biol. 7 (1997) 637-644.

[10] B. Henrissat, Enzymology of cell-wall degradation, Biochem. Soc. Transac. 26 (1998) 153-156.

[11] E.M.W. Chen, A.J. Mort, Nature of sites hydrolyzable by endopolygalacturonase in partially-esterified homogalacturonans, Carbohydr. Polym. 29 (1996) 129-136.

[12] J.A.E. Benen, H.C.M. Kester, J. Visser, Kinetic characterization of Aspergillus niger N400 endopolygalacturonases I, II and C, Eur. J. Biochem. 259 (1999) 577-585.

[13] E. Bonnin, A. Le Goff, G.J.W.M. van Alebeek, A.G.J. Voragen, J.-F. Thibault, Mode of action of Fusarium moniliforme endopolygalacturonase towards acetylated pectin, Carbohydr. Polym. 52 (2003) 381-388.
[14] G. André-Leroux, D. Tessier, E. Bonnin, Action pattern of Fusarium moniliforme endopolygalacturonase towards pectin fragments: comprehension and prediction, Biochim. Biophys. Acta 1749 (2005) 53-64.

[15] M.C. Ralet, M.J. Crépeau, E. Bonnin, Evidence for a blockwise distribution of acetyl groups onto homogalacturonans from a commercial sugar beet (Beta vulgaris) pectin, Phytochem. (2008) 1903-1909.

[16] G. van Pouderoyen, H.J. Snijder, J.A.E. Benen, B.W. Dijkstra, Structural insights into the processivity of endopolygalacturonase I from Aspergillus niger, FEBS Lett. 554 (2003) 462-466.

[17] Y. van Santen, J.A.E. Benen, K.-H. Schröter, K.H. Kalk, S. Armand, J. Visser, B.W. Dijkstra, 1.68-Å crystal structure of endopolygalacturonase II from Aspergillus niger and identification of active site residues by site-directed mutagenesis, J. Biol. Chem. 274 (1999) 30474-30480.

[18] L. Federici, C. Caprari, B. Mattei, C. Savino, A. Di Matteo, G. De Lorenzo, F. Cervone, D. Tsernoglou, Structural requirements of endopolygalacturonase for the interaction with PGIP (polygalacturonase-inhibiting protein), PNAS 98 (2001) 13425-13430.

[19] L. Holm, J. Park, DaliLite workbench for protein structure comparison, Bioinformatics 16 (2000) 566-567.

[20] L. DeLano, The case for open-source software in drug discovery, Drug Discov. Today 10 (2005) 213-217.

[21] N.A. Baker, D. Sept, S. JosepS, M.J. Holst, J.A. McCammon, Electrostatics of nanosystems: application to microtubules and the ribosome, Proc. Natl. Acad. Sci. U. S. A. 98 (2001) 10037-10041.

[22] T.J. Dolinsky, J.E. Nielsen, J.A. McCammon, N.A. Baker, PDB2PQR: an automated pipeline for the setup, execution, and analysis of Poisson-Boltzmann electrostatics calculations, Nucleic Acids Res. 32 (2004) 665-667.

[23] M. Tirion, Large amplitude elastic motions in proteins from a single-parameter, atomic analysis, Phys. Rev. Lett. 77 (1996) 1905-1908.

[24] M. Delarue, Y.-H. Sanejouand, Simplified normal mode analysis of conformational transitions in DNA-dependent polymerases: the Elastic Network Model, J. Mol. Biol. 320 (2002) 1011-1024.

[25] G. André, A. Buléon, M. Juy, N. Aghajari, R. Haser, V. Tran, Amylose chain behavior in an interacting context II-Molecular modelling of a maltopentaose fragment in the barley $\alpha$-amylase catalytic site, Biopolymers 49 (1999) 107-119.

[26] G. André, A. Buléon, R. Haser, V. Tran, Amylose chain behaviour in an interacting context III-Complete occupancy of the AMY2 barley $\alpha$-amylase cleft and comparison with biochemical data, Biopolymers 50 (1999) 751-762.

[27] E. Bonnin, A. Le Goff, R. Körner, J. Vigouroux, P. Roepstorff, J.-F. Thibault, Hydrolysis of pectins with different degrees and patterns of methylation by the endopolygalacturonase of Fusarium moniliforme, Biochim. Biophys. Acta 1596 (2002) 83-94.

[28] M. Kouwijzer, H. Schols, S. Pérez, Acetylation of rhamnogalacturonan I and homogalacturonan: theoretical calculations, in: J. Visser, A.G.J. Voragen (Eds.), Pectins and Pectinases, vol. 14, Elsevier Science, Amsterdam (The Netherlands), 1996, pp. 57-65.

[29] E. Bonnin, A. Le Goff, R. Körner, G.J.W.M. van Alebeek, T.M.I.E. Christensen, A.G.J. Voragen, P. Roepstorff, A. Caprari, J.-F. Thibault, Biochim. Biophys. Acta 1526 (2001) 301-309. 